\title{
Identification of Landslide Region Based on Topographic and Change Information
}

\author{
Minsi Ao ${ }^{1}$, Jianjun $\mathrm{Zhu}^{1 *}$, Changcheng Wang ${ }^{1}$ and Xiaoying $\mathrm{Yu}^{2}$ \\ ${ }^{1}$ School of Geosciences and Info-physics, Central South University, Changsha, \\ 410083, China \\ ${ }^{2}$ School of Civil Engineering and Architecture, Changsha University of Science and \\ Technology, Changsha, 410083, China \\ aominsi@csu.edu.cn,zjj@csu.edu.cn
}

\begin{abstract}
The accurate and rapid identification of landslide region is the basis for emergency disaster processing and analysis. The identification methods based on change information among multi-temporal remote optical sensing images are simple and intuitive. But its performance is remarkably limited by issues, such as unable to distinguish the changes according to the causes, improper processing strategy for impact of the adjacent pixels and the low efficiency. Thus, an approach based on digital elevation model and change information is presented in this paper. At first, the potential landslide region is calculated through slope information. And then the change information constrained by aspect information is used to identify and extract the final landslide regions. The experiment results show that, this approach can effectively distinguish the change information caused by city construction or landscaping planning. The extracted landslide regions are greatly consistent with interpretations. Meanwhile, it is effectively satisfy the demands for emergency disaster processing.
\end{abstract}

Keywords: Remote sensing; Change detection; Slope; Aspect; Landslide

\section{Introduction}

Natural hazards like landslides, which always occur with earthquake and floods, can result in enormous property damage and human casualties especially in mountainous regions such as Sichuan, southwestern in China [1]. Rapid and accurate identification and extraction of the landslide hazard region is the basis of analysis, assessment and predictions [2-4].

Different ways were developed to identify the landslide region, for instance, global positioning system [5], geotechnical investigation [6, 7], seismic retraction [8] and remote sensing related technology [9]. Compared to other technologies, the remote sensing is more popular due to less cost on time and labors, especially for large scale investigations. The main remote sensing technology to monitoring the landslide includes Lidar [10,11], synthetic aperture radar [12] and optical remote sensing images. Because of the rich multi-spectral information, the optical remote sensing images are one of the most popular information sources to identify, mapping and study the landslide hazard [13]. Some scholars tried to retrieve the landslide information from intensity, texture characteristics with single satellite image. Wang [14] and Yang [15] presented the automatic or semi-automatic landslide extraction approaches according to the condition that the quality or number of satellite images are not enough. While, with more and more images can be obtained from different satellites in space or sky, increasingly scholars choose to use the change information between multi- 
temporal images during landslide researches. Delacourt [16] measured the landslide velocity based on the correlation between the images from aerial and satellite images. Lu [17] and $\mathrm{Hu}$ [18] respectively detected the landslide region near mountain and rainforest area based on the object level. In these object-oriented methods, how to construct the rules for landslide object will directly determinate the performance of identification. Approaches based on change characteristic significantly improve the accuracy, but the incorrect identification due to nonlandslide factor is inevitable. To mitigate this and considering the strong correlation between the landslide and topography [19, 20], Yao [21], extract the landslide region with combination of single remote sensing image and elevation model (DEM). Barlow [22, 23] introduced the DEM into remote sensing landslide identification research. He used the DEM to constrain the object-level potential landslide for better results.

Since May in 2008, large amount of landslide occurred after the Wenchuan 7.9Ms earthquake, Sichuan Province, China. Meanwhile, the researches on the landslide with remote sensing also become the raises the scholar's interest. Li, et al., [24] extracted the landslide regions with the change information of texture features from the images before and after the Wenchuan earthquake. Gong [25] used the optical images during the Wenchuan earthquake to assess and analyze the landslide hazard statues in different scales. Liou [26] conducted the disaster assessment with the FORMOSAT-2 satellite images. The introduction of remote sensing technology played an important role on reducing the loss of property and lives.

Generally speaking, from the development of landslide identification approaches and application during Wenchuan earthquake, we can find that there are many issues worth to be considered further. First of all, the methods referred to machine-learning theory such as image classification, segmentation requires the large and remarkably different enough learning samples or priori knowledge. Secondly, it is difficult to distinguish the causes of change information, which leads to wrong identification. Thirdly, the impacts of the adjacent pixels or object are not properly considered in the traditional methods. For example, the equal weights during the spatial filtering are simple enough but not in accordance to real situations. Because of the issues mentioned above, the traditional methods to extract landslide region are principally used for low spatial-resolution and large scale remote sensing images. For highresolution ones, the visual interpretation is the most popular approach, while the efficiency of which cannot satisfy the needs of emergency hazard process. To solve these problems, an approach to extract the landslide region based on the topography and change information between multi-temporal high resolution remote sensing images is presented in this paper. It uses the slope information from topography to calculate the potential landslide region first, and then the change information constrained by aspects information is used to identify and extract the final landslide regions.

\section{Methodology}

The landslide identification approach based on topography and change information consists of four steps. First of all, it is the preprocessing of images. It includes the graying, precise registration, cropping and balancing the images and digital elevation models (DEM). Secondly, the threshold for potential landslide region is to be calculated from the slope and aspects information derived by DEM. Then, the pixel-level change characteristics of multitemporal remote sensing images is to be obtained through spatial filtering constrained by aspects information and thresholding. At last, with confusion of potential region and multitemporal change characteristic the final landslide region can be retrieved. During the procedures, the key problems concentrate on setting of thresholds for potential region and change characteristic, and the spatial filtering constrained with aspects information. 


\subsection{Potential Landslide Region}

DEM is one the most important data for spatial and terrain analysis in geographic information system. There are many ways for acquisitions of DEM, such as elevation measurements, synthetic aperture radar, satellites stereo images and so on. Due to the different spatial resolution between DEM and remote sensing images, the cubic spline interpolation is often used to resample them to be equivalent. The slope and aspect derived from the DEM are two important terrain parameters especially for analyze and assess the landslide hazards [27, 28]. During the extraction of potential region, how to select the threshold is critical to the final results. The relationship between the landslide and slope is correlated with geological environments within the certain area closely. Comprehensively, the different landslides often correspond to different critical slope. If the geological information is complete and accurate, the slope threshold can be determined by them or the experiences. Otherwise, an estimation of the slope threshold which based on the histogram and its minimum is presented in this paper. In this method, based on the histogram of slope value, the local minimum which is the most close to mean slope value within interval [30-60] degrees can be tested individually to be the threshold. With this strategy, the obtained thresholds are usually at the bottom of the slope, which can extract the most potential hazard regions.

\subsection{Change Characteristic Information}

Extract the landslide region through the change characteristic from multi-temporal images is one of the most rapid and intuitive method. At present, the principal indexes to measure the changes are still the differences or ratios. In the traditional change detection methods based on pixels, the weights of adjacent pixels usually are treated equivalent or even not treated at all. With consideration of the strong correlation between the landslide and aspect, an anisotropic spatial filtering algorithm based on the aspect is presented. The filtering weights in this approach are determined by the aspects. In this algorithm, the aspects are divided into eight intervals respective to four directions. When the aspect is north to east, west-south to north-east situations, and with windows length setting to one, the weights of adjacent pixels are shown in Figure 1.

\begin{tabular}{|l|l|l|}
\hline 1 & 2 & 1 \\
\hline 1 & 2 & 1 \\
\hline 1 & 2 & 1 \\
\hline
\end{tabular}

(a) North-south

\begin{tabular}{|l|l|l|}
\hline 1 & 1 & 2 \\
\hline 1 & 2 & 1 \\
\hline 2 & 1 & 1 \\
\hline
\end{tabular}

(b) Northeast-southwest

\section{Figure 1. The Weights Strategy of the Adjacent Pixels of Spatial Filtering}

As is shown in Figure 1, when the aspect is within the interval [-22.5 to 22.5] or [157.5 to 202.5] degrees, the north-south weights strategy will be adopted. Meanwhile, if the aspect is within [22.5 to 45] or [202.5 to 225] degrees, the northeast to southwest strategy should be chosen. This strategy can be easily extended to other directions. At the same time, the noises aliasing in the change information can also be mitigate through setting different window length. After acquisition of the whole change characteristic map, there are a lot of ways to determine the threshold. In order to mitigate the impacts of subjective factors and dependence 
on prior knowledge, in this algorithm the threshold setting is based on the mean value and standard deviation of the whole change characteristic map, which is shown as equation.

$$
\text { thr }{ }_{-} c=\operatorname{mean}(c(i, j)) \pm n \cdot \operatorname{std}(c(i, j))
$$

In Eq. (1), thr $r_{-} c$ is the threshold for change characteristic, $c(i, j)$ is the difference between the multi-temporal images, $n$ is the change threshold coefficient. In the traditional method, the change threshold coefficients are usually setting to $0,0.1$ or even larger. While in this approach, it is often setting to within the interval [-0.3 to -0.1] to include much more change information. Finally, the regions which are both located in the potential area and exhibits enough change information would be deemed as the landslide region.

\section{Experiments and Analysis}

After the Wenchuan earthquake in China, the research institutes and companies quickly organized the acquisition of amount of remote sensing images. In this section, the area of interest (AOI) from part of the Wenchuan County is selected as the study case. The basic statues of AOI are shown in Figure 2.

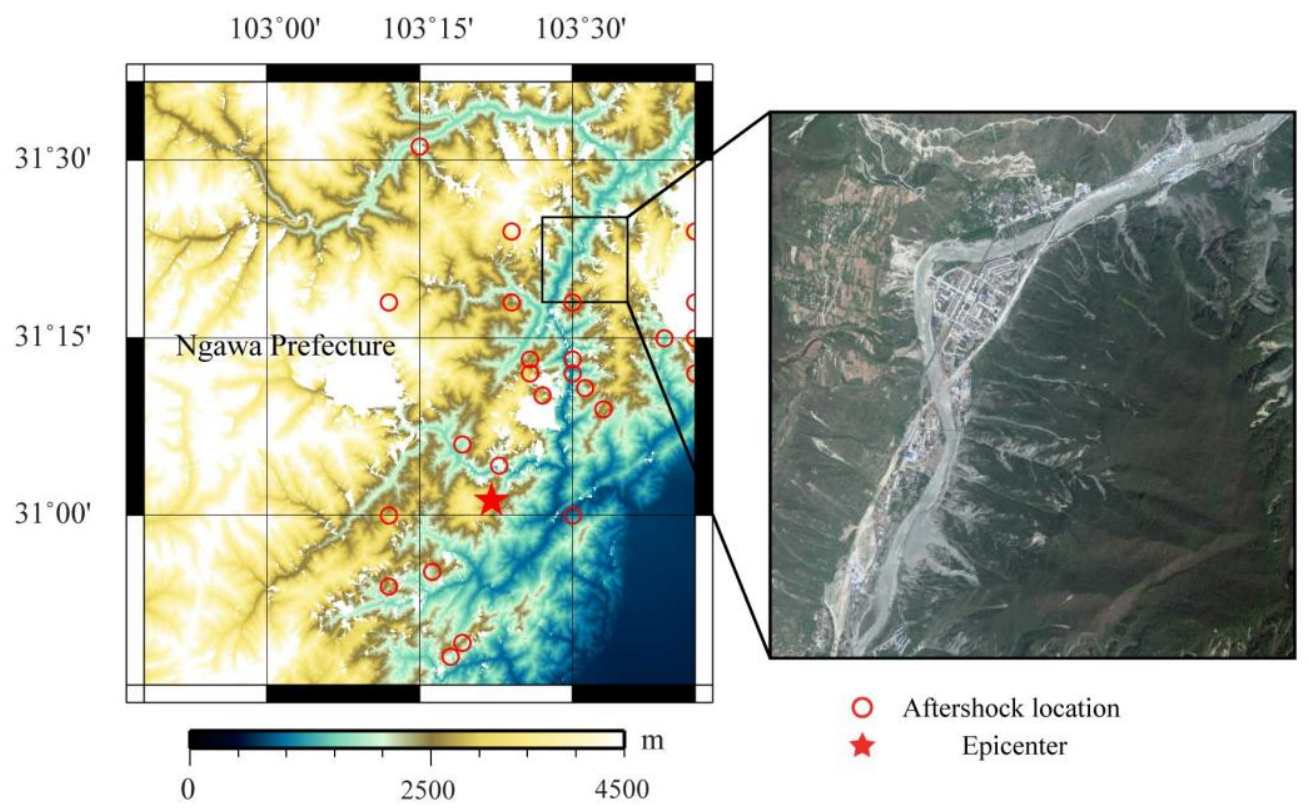

Figure 2. Basic statues of Wenchuan County and AOI

Figure 2 shows the location and elevation condition of Wenchuan County, which is southeastern parts of the Ngawa Zangzu Qiangzu Autonomous Prefecture, Sichuan Province. The Wenchuan County is in the transition zone from the Tibet Plateau to Western Sichuan Plain. There are Minshan, Longmenshan and Qianglaishan mountains respectively in north, south and west. The terrain decrease significantly from the northwest to southeast, the mountains are mainly at about more than 4000 meters above sea level. The regional neotectonism showed a sharp increase in the crust and faulting. Three major faults (Qingchuan-maowen fault, Beichuan-yingxiu fault and Jiangyouguanxian fault) lay out in northeast-southwest direction. Thus, the earthquakes occur frequently in this region. As is shown in the left of Figure 2, the Wenchuan earthquake 
M7.9s and the aftershocks are also denoted respectively as the star and cycles. The area in black box, which is in the right part of Figure 2, is the AOI, Miansi town. The left part of Figure 2 is an ortho image from worldview satellite. The Min River, which is deep, rushing and surrounding by soil covered inface, is the main water system in this area. Although the image was taken in April, 2011, the damages caused by landslide can still be found along the slope next to the Min River. The landslides are principally developed in the bottom of the shore on both side of the valley slope. The shallow landslides are much more than the deep ones. With comparison and analysis among the visual interpretation, method from [24] and approach presented in this paper is conducted.

\subsection{Data Collection and Preprocess}

In this case study, the image before landslide was collected on 9th, September in 2005. It is part of the panchromatic IKONOS satellite images. The image after landslide was collected on 23rd, May in 2008, which is 12 days after the Wenchuan earthquake. It is from the panchromatic Quickbird satellite image. The free DEM data are from the Shuttle Radar Topography Mission (SRTM). With cubic spline interpolation, all the images and DEM are turned into 647 rows by 746 columns. After preprocess mentioned above, the images and DEM are shown as Figure 3.

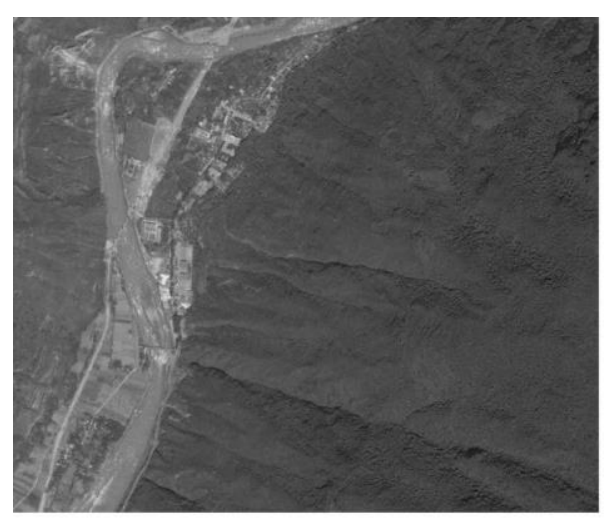

(a) pre-earthquake

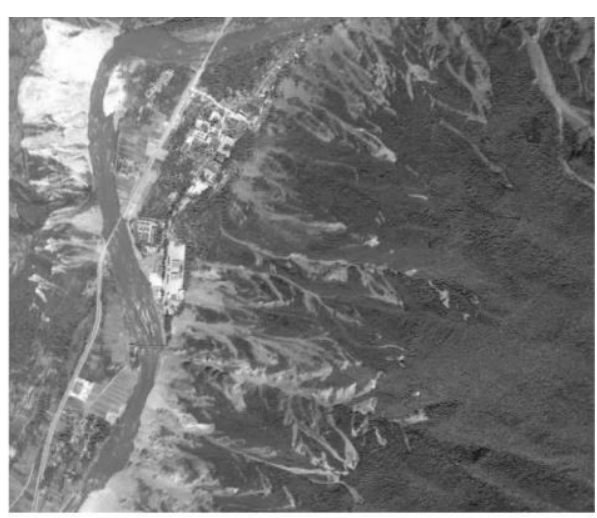

(b) post-earthquake

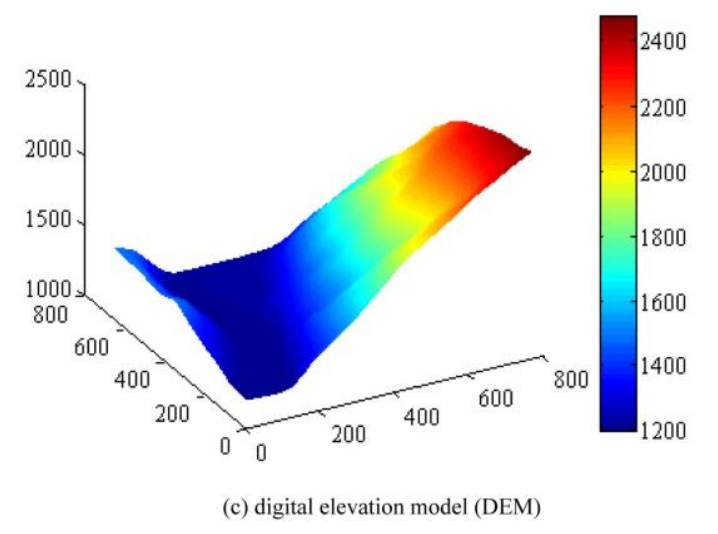

Figure 3. The Preprocessed Satellite Images and DEM

As is shown in Figure.3, the Miansi town locates in the northwest part of the images. The Min River runs from the north to west and then obviously turns back to the 
southwest directions. For the whole AOI, the elevation of the northeast and southwest are much higher than the middle. Meanwhile, the landslides are also distributed on both side of the Min River.

\subsection{Potential Landslide Region of AOI}

In this study area, the geological information is much enough to figure out that the landslides usually happened above 50 degrees. The statistics of the slope distribution which derived from the DEM is shown in Figure 4.

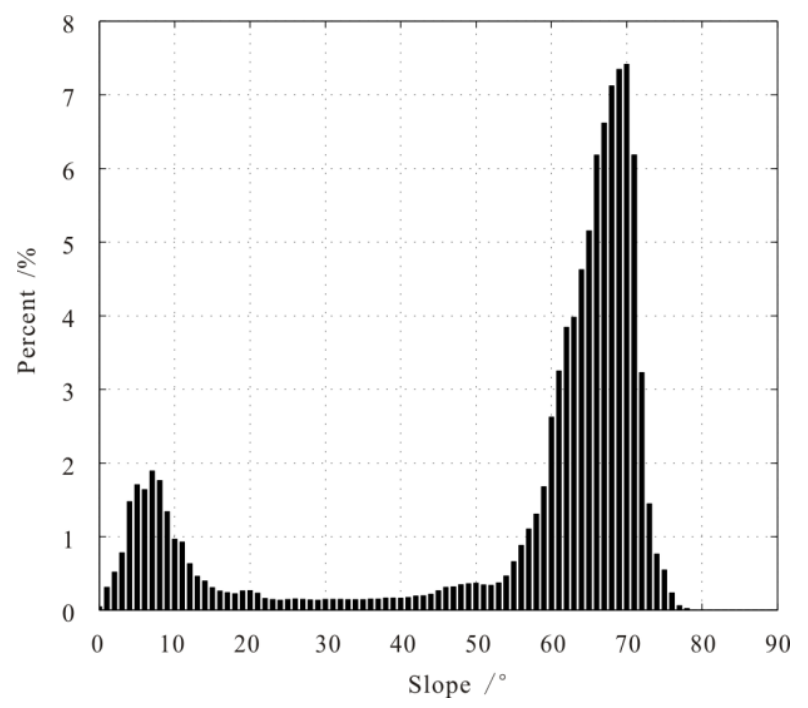

Figure 4. Statistics of the Slope Distribution

As is shown in Figure 4, the slopes of the study area distribute from 0 to 79 degree, and the mean value of which is about 54.9 degree. With the determination strategy of the slope threshold mentioned above, the threshold can be setting to 55 degrees. The extracted potential landslide region is shown in Figure 5.

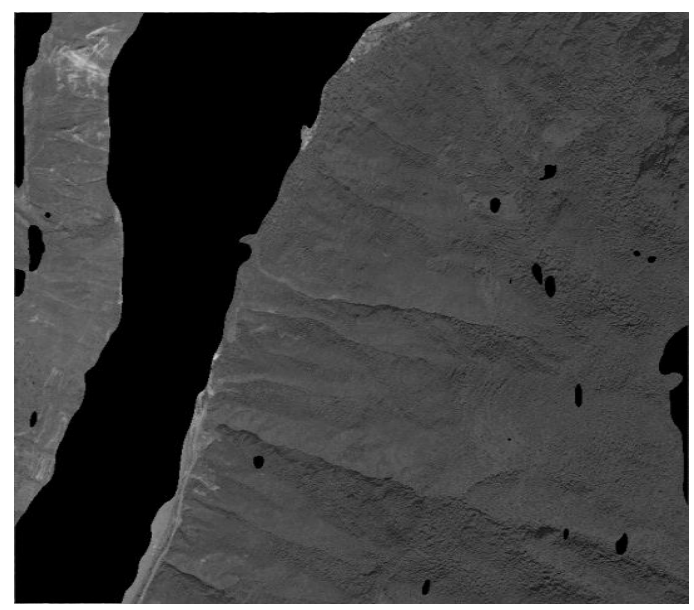

Figure 5. The Potential Landslide Region Extracted with Thresholding 
In Figure 5, the areas not black are the potential landslide regions. It is obvious that the extracted potential landslide regions are mainly around slopes of the sides of Min River. Due to the whole terrain characteristic, the southeast, northwest parts in the images are selected as the potential regions. Meanwhile, the Miansi town which locates in the middle of the central north is not judged to be the potential ones because of its lower slope.

\subsection{The Change Characteristics Information}

During the extraction of change characteristic, the filtering window length and threshold are two of the most important parameters. With the potential landslide region mentioned above, when the change threshold coefficients are respectively setting as $n=-0.4,-0.3, \ldots, 0.2$, and the window length varies from 5 to 13 , the correction ratio between the extracted landslide region and visual interpretations are shown as Table 1.

Table 1. Correction Ratio from Different CTC and Window Length

\begin{tabular}{ccccccccc}
\hline \multirow{2}{*}{$w$} & thr_c & -0.4 & -0.3 & -0.2 & -0.1 & 0 & 0.1 & 0.2 \\
\hline \multirow{3}{*}{$\mathrm{w}=5$} & $R$ & $\mathbf{8 1 . 5}$ & 84.6 & 86.1 & 86.4 & 85.8 & 84.7 & 83.7 \\
& $r 1$ & 70.5 & 78.6 & 85.8 & 90.4 & 92.8 & 93.7 & 94.2 \\
& $r 2$ & 88.4 & 87.5 & 86.3 & 85 & 83.7 & 82.2 & 81 \\
$\mathrm{w}=7$ & $R$ & 82 & 85.2 & 86.7 & 86.9 & 86.2 & 85 & 83.8 \\
& $r 1$ & 70.6 & 78.9 & 85.8 & 90.2 & 92.3 & 93.2 & 93.7 \\
& $r 2$ & 89.5 & 88.4 & 87.1 & 85.7 & 84.2 & 82.7 & 81.2 \\
$\mathrm{w}=9$ & $R$ & 82.5 & 85.6 & 87.1 & $\mathbf{8 7 . 4}$ & 86.3 & 85.1 & 83.9 \\
& $r 1$ & 70.8 & 79 & 85.7 & 89.9 & 91.8 & 92.5 & 93.1 \\
& $r 2$ & 90.3 & 89.1 & 87.6 & 86.2 & 84.5 & 82.9 & 81.4 \\
$\mathrm{w}=11$ & $R$ & 82.6 & 85.8 & 87.2 & 87.2 & 86.2 & 85.1 & 83.9 \\
& $r 1$ & 70.8 & 78.9 & 85.3 & 89.3 & 90.9 & 91.8 & 92.5 \\
& $r 2$ & 90.7 & 89.5 & 88 & 86.5 & 84.6 & 83 & 81.5 \\
$\mathrm{w}=13$ & $R$ & 82.7 & 85.9 & 87.2 & 87.1 & 86.1 & 85 & 83.8 \\
& $r 1$ & 70.7 & 78.8 & 85.1 & 88.5 & 90.1 & 91.1 & 91.8 \\
& $r 2$ & 91 & 89.8 & 88.3 & 86.5 & 84.8 & 83.1 & 81.5 \\
\hline
\end{tabular}

In Table.1, the $R, r 1$ and $r 2$ are respectively the whole accuracy, first and second kind of accuracy. The first and second kind of accuracy is defined as $r=N_{r} / N_{t o t}$. The $N_{r}$ denotes the number of landslide or non-landslide pixels relative to the results from visual interpretations, while the $N_{t o t}$ denotes the whole landslide or non-landslide ones. With $R, r 1$ and $r 2$, the analysis can be conducted from aspect of whole, landslide and non-landslide. It is can be seen that within the whole threshold interval, the minimal overall accuracy is $81.5 \%$ which is according to threshold -0.4 and window length 5 . While the maximal overall accuracy reaches $87.4 \%$ when the threshold set to be -0.1 and window length to be 9 . The mean overall accuracy is about $85.2 \%$. For setting of the change threshold coefficient, when it increases from -0.4 to 0.2 , no matter how the window length to vary, the maximal overall accuracy is reached at -0.1 or -0.2 . While in the traditional methods, it usually is set to be 0 or 0.1 . It indicates that, with assumption the same overall accuracy reached, the more weak change characteristic can be detected by approach presented in this paper because of the potential landslide procedures. 
Further, it is easy to figure out that the first and second kind accuracy are respectively monotonically increasing and decreasing trends. The reasons for the monotonical trends can be explained as, when the change threshold increases, the extracted change characteristic is more remarkable but less in number, it leads to the increasing of first kind accuracy. At the same time, part of the landslide pixels whose change characteristic is weak may be identified as the non-landslide. And it leads to the decreasing of the accuracy for landslide. Both of the two reasons lead to the respective increasing and decreasing trends. These trend indicates that the capability on identifying the landslide and non-landslide increases and decreases respectively to the change threshold coefficients. Therefore, in practical situations, the balance should be kept between the identification on both landslide and non-landslide. For filtering window length parameter, it gives a weaker impact on the accuracy. For $R, r 1$ and $r 2$, the respective most variation are $1.2 \%, 2.7 \%$ and $2.6 \%$. However, the number of the sub-regions will be influenced greatly by the filtering which is determined by the window length. The number of landslide sub-regions according to different window lengths is shown in Figure 6.

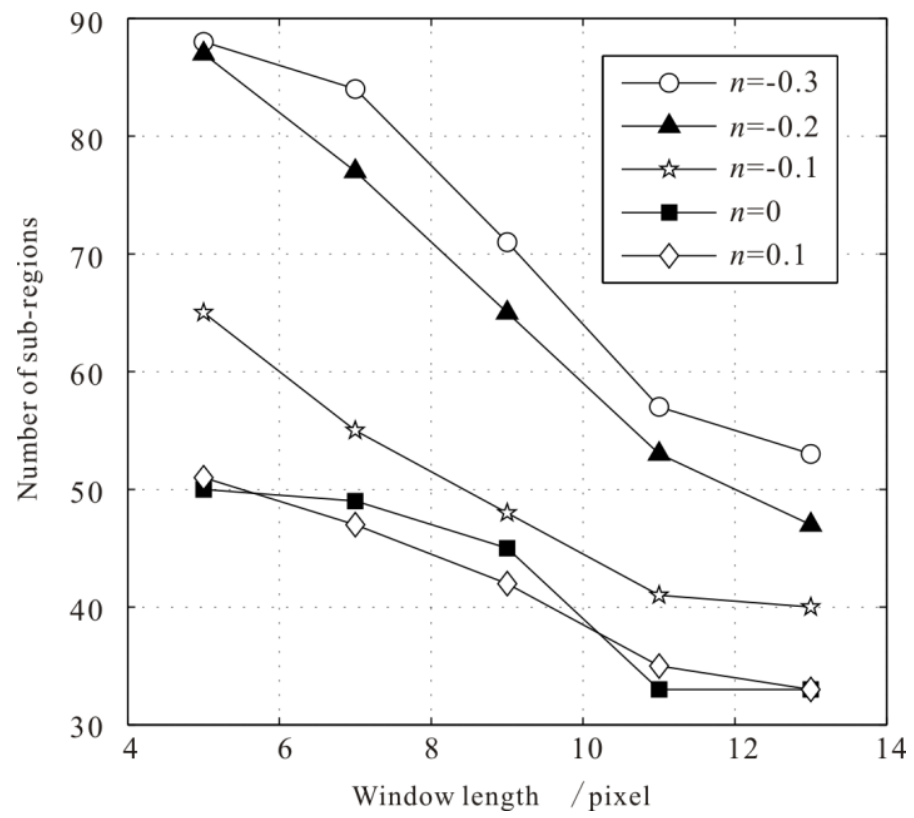

Figure 6. The Relationship between the Filtering Window Length and Number of Landslide Sub-regions

In Figure 6, due to the similarities, only the curves of variation of the number of subregions when $n=-0.3, \ldots, 0.1$ are plotted. It is obvious to figure out that, regardless of change threshold coefficient variation, the number of landslide sub-regions is decreasing when the window length increases. This kind of phenomenon is in consistent with the traditional filtering no doubt. Meanwhile, when the change threshold coefficient increases, the fewer pixels would be identified as landslide relatively, which will leads to the decreasing of number of the landslide sub-regions. With further comparison on the five curves, when the change threshold coefficient is setting to -0.3 or -0.2 , the decreasing speed is faster, while $-0.1,0$, or 0.1 , the speed is nearly the same. Compared to the formers, the number of the landslide sub-regions is decreasing to be 
steady, which indicates that the parameters are become stable and the convergence. It should be noted that it is the not the best but the most conservative results. Thus, when the filtering window length is set to be 9 or 11 is prone to obtain the better results. In this case study, the change threshold coefficient and filtering window length are respectively setting to -0.1 and 9 .

\subsection{Results and Analysis}

With the potential landslide region and change characteristic, the final landslide region can be retrieved through simple area confusions. The comparison on validity and efficiency will be discussed based on the results among three approaches. The results from this approach, approach referred [24], and visual interpretation are shown in Figure 7.

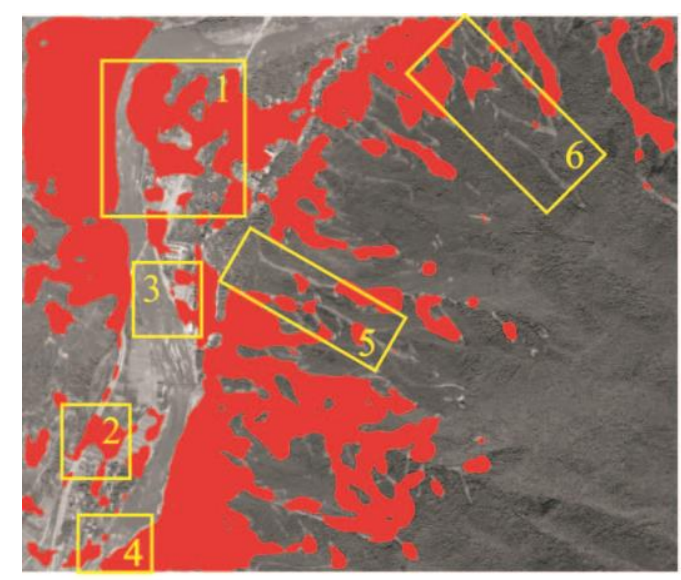

(A) results from approach of reference

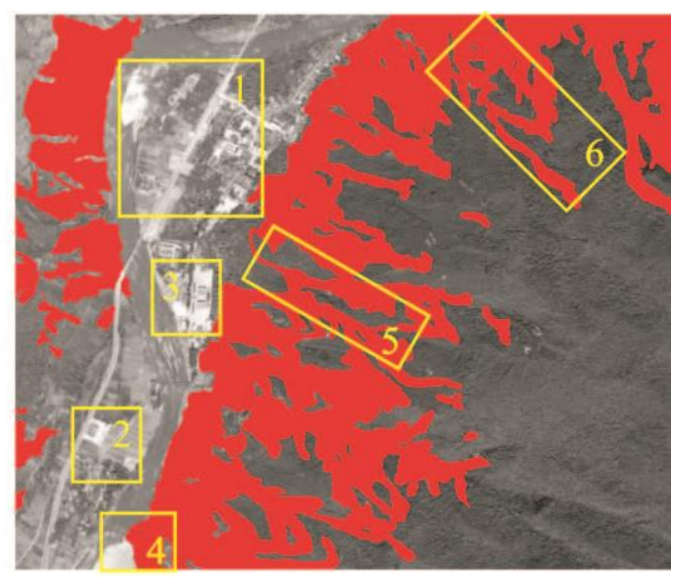

(B) results from visual interpretation

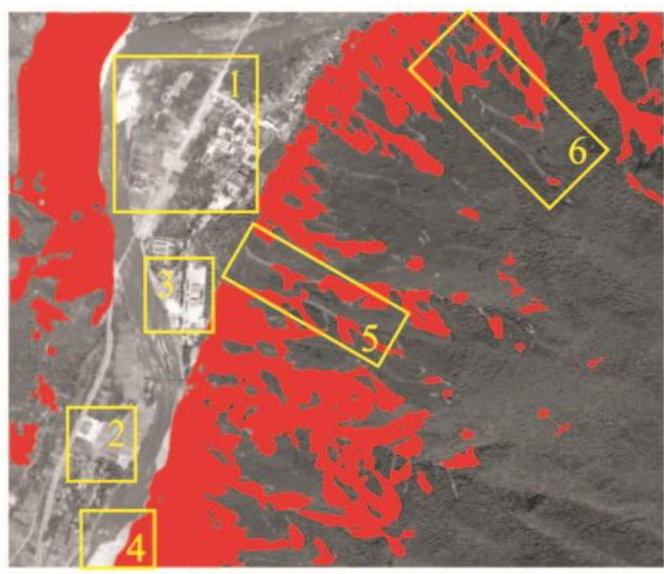

(C) results from approach of this paper

\section{Figure 7. Landslide Region Extracted from this Three Approaches}

In Figure 7, the sub-figure (A) is from approach from [24]. It extracted the landslide region only based on the characteristic of image texture. The sub-figure (B) is extracted by visual interpretation. The sub-figure (c) is results of approach presented in this paper. In the following discussion, the three approach will be referred as first, second and third 
approach. With comparison between the sub-figures (A) and (C) in Figure 7, the remarkable differences can be found. For example, in area (1), (2), (3) and (4), they are indentified as landslide by first approach but not by third one. The further detail interpretation indicates that, the area (1) is actually belonging to the town. The external materials showed that the only damage occurred just in the southeast part of the town. At the same time, it is worthy to note that, the change definitely happened in the middle of town between the temporal remote sensing images. But they are mainly caused by the replanning and reconstruction of the buildings, roads and bridges. These kinds of the change information are incorrectly recognized as the landslides in the first approach. The more obvious case occurred are from the area (2) and (3), the rebuilding houses cause the wrong recognitions. The situation in area (4) is more complicated. For one hand, this area is next to the slope in southeast, it is damaged by landslides. For another hand, the other change information was caused by the rising of the river level due to the rainy season. The first approach identifies that the whole area (4) to be landslides incorrectly. The minor differences between the sub-figure (B) and sub-figure (C) show that they are nearly equivalent. But the minor differences between them are still worthy to be discussed. For instance, the area (5) and (6) are recognized as landslides by visual interpretation, but not by the first and third approach. If we check the images carefully, it can be found that these smaller landslides are formed not during the time between multi-temporal images. It does not construct the effective change characteristics successfully. Therefore, the first and third approaches based on the change information cannot identify them while the visual interpretation does. To these kinds of former landslides, it is the problem of all the approach based on change characteristics. To computational efficiency, under the hardware environments as 3.3G CPU, 2G RAM and platform Matlab2010b in windows, the computational time are respectively $13.4 \mathrm{~s}, 10.1 \mathrm{~s}$ and about 20 minutes (with MAPGIS remote sensing modules). The difference efficiency between the first and third approach is mainly from the processing of DEM. Both two of them can meet the hazard emergency process applications. Overall, the approach presented in this paper can effectively extract the landslide regions and eliminate the change information cased by reconstruction or replanning. The results are basically consistent with visual interpretation and the computational efficiency can satisfy the hazard emergency process applications.

\section{Conclusion}

Accurate and rapid identification and extraction of the landslide regions plays an important role for hazard analysis, assessment and prediction. To solve the issues such as dependency of prior knowledge, improper strategy for impacts of adjacent pixels and computational efficiency in traditional approaches, an approach based on the topography and change characteristic is presented in this paper. It uses the slope information derived from topography to calculate the potential landslide region first, and then the change information constrained by aspects information is used to identify and extract the final landslide regions. The experiments and results show that, this approach can effectively identify the landslide regions and eliminate the change information cased by reconstruction or replanning. The results are basically consistent with visual interpretation and the computational efficiency can satisfy the hazard emergency process applications. Meanwhile, it can be seen during the case study that there are still issues that, for example, how to recognize the landslides formed before 
the periods of multi-temporal images and realize the automate parameter setting based on the images themselves, are worth consideration in future.

\section{Acknowledgements}

This work was supported in part for the author by the National High Technology Research and Development Program of China (863 Program) (No.2012AA121301), the National Basic Research Program of China (973 Program) (No. 2013CB73303), National Natural Science Foundation (41371335), China Postdoctoral Sciences Foundation, Hunan Province Postdoctoral Scientific Research Fund project, and Central South University Postdoctoral Sciences Foundation.

\section{References}

[1] C. Xu, F. Dai and X. Xu, "Wenchuan Earthquake-induced Landslides: an Overview", Geological Review, vol. 56, no. 6, (2010), pp. 860-874.

[2] J. Zhang, "3S-aided Landslide Hazard Monitoring and Modeling", Engineering of Surveying and Mapping, vol. 14 , no. 2, (2005), pp. 1-5.

[3] J. J. Zhu, X. L. Ding and Y. Q. Chen, "Dynamic Landsliding Model with Integration of Monitoring Information and Mechanic Information", Acta Geodaetica et Cartographica Sinica, vol. 32, no. 3, (2003), pp. 261-266.

[4] C. F. Mahler, E. Varanda and L. C. D. de Oliveira, "Analytical Model of Landslide Risk Using GIS", Open Journal of Geology, vol. 2, no. 3, (2005) , pp. 182-188.

[5] P. Zhou, B. Zhou, J. Guo, D. Li, Z. Ding and Y Feng, "A Demonstrative GPS-aided Automatic Landslide Monitoring System in Sichuan Province", Journal of Global Positioning System, vol. 4, no. 1-2, (2005), pp. 184-191.

[6] P. D. Epada, G. Sylvestre and T. C. Tabod, "Geophysical and Geotechnical Investigations of a Landslide in Kekem Area, Western Cameroon”, International Journal of Geosciences, vol. 3, no. 4, (2013), pp. 780-789.

[7] V. Lapenna, P. Lorenzo, A. Perrone, S. Piscitelli, E. Rizzo and F. Sdao, "2D Electrical Resistivity Imaging of Some Complex Landslides in Lucanian Apennine Chain, Southern Italy”, Geophysics, vol. 70, no. 3, (2005), pp. 11-18.

[8] T. Glade, P. Stark and R. Dikau, "Determination of Potential Landslide Shear Plane Depth Using Seismic Refraction- A Case Study in Rheinhessen, Germany", Bulletin of Engineering Geology and the Environment, vol. 64, no. 2, (2005), pp. 151-158.

[9] F. Mantovani, R. Soeters and C. J. Van Westen, "Remote sensing techniques for landslide studies and hazard zonation in Europe", Geomorphology, vol. 15, no. 3-4, (2005), pp. 182-188.

[10] N. F. Glenn, D. R. Streutker, D. J. Chadwick, G. D. Thackray and S. J. Dorsch, "Analysis of LiDAR-derived topographic information for characterizing and differentiating landslide morphology and activity", Engineering Geology, vol. 73, no. 1-2, (2006), pp. 131-148.

[11] J. McKean and J. Roering, "Objective landslide detection and surface morphology mapping using highresolution airborne laser altimetry", Geomorphology, vol. 57, no. 3-4, (2004), pp. 331-351.

[12] C. Squarzoni, C. Delacourt and P. Allemand, "Nine years ofspatial and temporal evolution of the La Valette landslide observed by SAR interferometry”, Engineering Geology, vol. 68, no. 1-2, (2003), pp. 53-66.

[13] J. E. Nichol, A. Shaker and M. Wong, "Application of High-resolution Stereo Satellite Images to Detailed Landslide Hazard Assessment”, Geomorphology, vol. 76, no. 1-2, (2006), pp. 68-75.

[14] S. B. Wang, D. M. Zhang, B. Luo and C. Y. Zhang, "Semi-automatic Landslide Extraction for Remote Sensing Image Based on Spectral Matting”, Computer Engineering, vol. 38, no. 2, (2012), pp. 195-197.

[15] S. Yang, F. Xie, H. Han and G. Feng, "Automatic Extraction of Shallow Landslildes Based on SPOT-5 Remote Sensing Images", Science of Surveying and Mapping, vol. 37, no. 1, (2012), pp. 71-88.

[16] C. Delacourt, P. Allemand, B. Casson and H. Vadon, "Velocity field of the 'La Clapière' landslide measured by the correlation of aerial and QuickBird satellite images", Geophysical Research Letters, vol. 31, no. 15, (2004), pp. L15619.

[17] P. Lu, A. Stumpf, N. Kerle and N. Casagli, "Object-Oriented Change Detection for Landslide Rapid Mapping”, IEEE Geoscience and Remote Sensing Letters, vol. 8, no. 4, (2011), pp. 701-705.

[18] D. Y. Hu, J. Li, W. J. Zhao, C. Y. Zhang and G. X. Peng, "Object-oriented Landslide Detection from Remote Sensing Imageries with High Resolution”, Journal of Natural Disasters, vol. 17, no. 6, (2008), pp. 42-46.

[19] K. Jones, "A comparison of algorithms used to compute hill slope as a property of the DEM", Computers and Geosciences, vol. 24, no. 4, (1998), pp. 315-325. 
[20] F. Guo, N. Yang and H. Meng, "Application of the Relief Amplitude and Slope Analysis to Regional Landslide Hazard Assessments”, Geology in China, vol. 35, no. 1, (2008), pp. 131-143.

[21] X. Yao, Y. Zhang, X. Wang and T. Xiong, "Automatic Hierarchical Approach to Detecting Debris Flows, Landslideds and Avalanches by Combination of Multi-spectral RS Imagery and DEM Derivatives", Geological Bulletin of China, vol. 27, no. 11, (2008), pp. 34-39.

[22] J. Barlow, Y. Martin and S. E. Franlin, "Detecting Translational Landslide Scars Using Segmentation of Landsat ETM+ and DEM data in The Northern Cascade Mountains, British Columbia", Canadian Hournal of Remote Sensing, vol. 29, no. 4, (2003), pp. 510-517.

[23] J. Barlow, S. Franklin and Y. Martin, "High spatial resolution satellite imagery, DEM derivatives, and image segmentation for the detection of mass wasting processes", Photogrammetric Engineering and Remote Sensing, vol. 72, no. 6, (2006), pp. 687-692.

[24] S. Li, Y. Q. Li and Y. L. An, “Automatic Recognition of Landslides Based on Change Detection”, Remote Sensing Information, no. 1, (2010), pp. 27-31.

[25] J. Y. Gong, H. G. Sui, K. M. Sun, G. R. Ma and J. Y. Liu, "Object-level Change Detection Based on Fullscall Image Segmentation and Its Application to Wenchuan Earthquake", Science in China Series E: Technological Sciences, vol. 51, no. S2, (2008), pp. 110-122.

[26] Y. A. Liou, S. K. Kar and L. Y. Chang, "Use of High-resolution FORMOSAT-2 Satellite Images for Postearthquake Disaster Assessment: A Study Following 12 May 2008 Wenchuan Earthquake", International Journal of Remote Sensing, vol. 31, no. 13, (2010), pp. 3355-3368.

[27] X. J. Liu, J. Y. Gong and Q. M. Zhou, "A Study of Accuracy and Algorithms for Calculating Slope and Aspect Based on Grid Digital Elevation Model (DEM)", Acta Geodaetica et Cartographica Sinica, vol. 33, no. 3, (2004), pp. 258-263.

[28] J. Gao, "Identification of topographic settings conducive to landsliding from DEM in Nelson County, Virginia, USA", Earth Surface Processes and Landforms, vol. 18, no. 7, (1993), pp. 579-591.

\section{Authors}

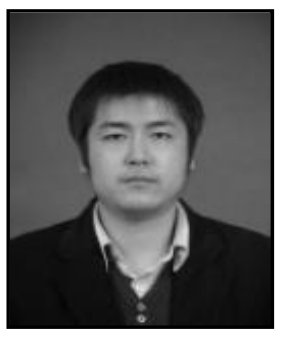

Minsi Ao, received his B.S. and M.S. degree in Information and communication engineering in June 2007, June, 2009 and Ph.D. degree in Cartography and Geographic Information Engineering in June 2012, from China University of Geosciences, China. He is currently conducting the postdoctoral research in Central South University, China. His current research interest includes the remote sensing, GNSS signal processing and its application in geosciences, especially for geological hazard.

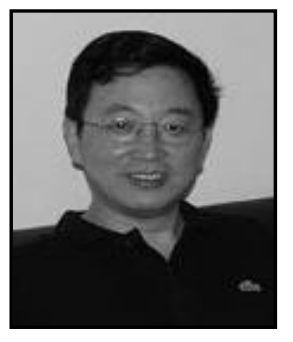

Jianjun Zhu, received the B.S., M.S. and Ph.D. degree in Surveying and mapping from Central South University, Changsha, China, in 1982, 1985 and 1998. He finished the postdoctoral research in Laval University in Canada. He is now the tenured full professor at the School of geosciences and info-physics, Central South University. His current research interest includes the remote sensing, GNSS signal processing and its application in surveying and mapping sciences.

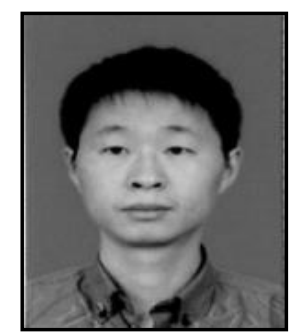

Changcheng Wang, received the Ph.D. degree in Photogrammetry and remote sensing from State Key Laboratory of Information Engineering in Survey, Mapping and Remote Sensing, Wuhan University in June 2009, Wuhan, China. He is the associate professor in school of geosciences and info-physics in Central South University. His current research interest includes the image processing, SAR/PolSAR image processing and its applications. 


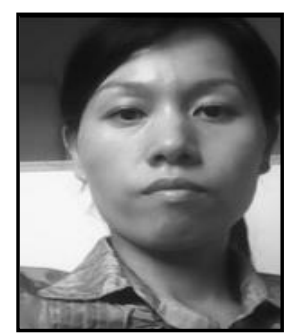

Xiaoying Yu, received her Ph.D. degree in Geodesy and Surveying Engineering from School of Geosciences and Info-physics, Central South University in December 2012. She is now the senior lecturer in Changsha University of Science and Technology. Her current research interest includes the image processing, Interferometric Synthetic Aperture Radar image processing and its applications. 
International Journal of Signal Processing, Image Processing and Pattern Recognition Vol.7, No.1 (2014) 